\title{
Spasticity patterns of hand muscles and botulinum toxin therapy application in patients with cerebral palsy with upper limb involvement
}

\section{Author affiliation:}

Ol'ga Andreevna Klochkova, pediatrician of the medical rehabilitation department for children with neurological disorders at the research institute of preventive pediatrics and medical rehabilitation of the SCCH

Address: 2, Lomonosovskiy Av., Moscow, 119991, tel.: +7 (499) 134-01-69, e-mail: Klochkova oa@nczd.ru

Article received: 21.05 .2013 . Accepted for publication:

Botulinum toxin therapy is an effective and safe method of treatment of local spasticity in patients with cerebral palsy (CP). Calculation of botulinum toxin A (BTA) dosage based on the spasticity patterns and functional capabilities of the patient proved effective for the hypertonic lower limb muscle spasm treatment and is being applied to BTA injections in hand muscles more often. The article presents contemporary scientific data and results of the original study of BTA injections efficacy for pathologic tension reduction in hand muscles of 52 patients with CP. The authors give detailed description of the upper limb spasticity patterns, their frequency and role in the pathological movement pattern formation. The authors propose BTA dosage calculation for the functional segments of upper limbs, which allows minimizing the total amount of the administered drug and avoiding excessive weakness. The authors have also conducted a followup analysis of changes in hand muscle tone for the period of 6 months after the first BTA injection, compared results of botulinum toxin therapy at various clinical forms of CP and given recommendations on the optimum duration of the follow-up period.

Keywords: cerebral palsy, spastic diplegia, hemiparetic form, spasticity, spasticity patterns, botulinum toxin therapy, botulinum toxin A, ultrasonic control, hand, rehabilitation, Ashworth scale, muscle weakness scale.

\section{Introduction}

Botulinum toxin A (BTA) has been used in the world pediatric practice for ca. 20 years [1]. In Russia, the drug was approved for treatment of movement disorders in 1999. Botulinum therapy has been included into the national standards of cerebral palsy (CP) treatment since 2004. By that time, experience of effective BTA use for reduction of pathological muscular tonus both in lower and upper extremities had been accumulated [2-6], which is why there appeared more official indications to botulinum therapy for children in a range of countries [7]. In Russia, the registered indications to the BTA use in children over 2 years of age are "dynamic foot deformation due to spasticity in children with CP" (for drug Dysport) and "focal spasticity associated with dynamic "tip foot deformation" (for drug Botox). Therefore, only the 2 mentioned drugs are approved for use in children in Russia. In case of muscular spasticity of upper extremities in children with $\mathrm{CP}$, they are used off-label, by agreement with the local ethics committees of medical establishments and if the patients' representatives give their informed consent.

Systematic reviews and meta-analyses of previous works showed that "BTA injections increase efficacy of conservative methods of upper extremity function development at CP to a high significance extent while reducing motor deficiency and improving functional result and achievement of the previously set rehabilitation goals. Botulinum therapy appeared to be insufficiently effective as the only method of treatment in comparison with placebo or in absence of any other treatment. Botulinum therapy must be supplemented by further rehabilitation 
measures $[6,8]$.

BTA may be injected into muscles of upper extremities at CP in order to:

- reduce spasticity and eliminate dynamic deformation;

- improve a particular function of the extremity (shoulder rotation, flexion/extension of elbow, pronation/supinations of forearm, hand grip etc.);

- establish interaction of agonist and antagonist muscles;

- facilitate further rehabilitation measures;

- improve appearance of the extremity and the patient's self-appraisal [9].

Botulinum therapy may be used for palliative purposes even in case of intense malfunction of upper extremities: in order to reduce pain and facilitate medical care [10, 11].

As with lower extremities, target muscles for BTA injection in upper extremities are selected on the basis of individual appraisal of motor function deficiency and spasticity patterns together with identification of key muscles and their role in the child's motor disorders and determination of immediate and long-term therapy goals [6].

Identification of separate patterns of the relatively permanent and most widespread combinations of motor disorders caused by involvement of certain muscle groups in the pathological process helps to standardize selection of target muscles and further rehabilitation aims [9, 12-16]. Spasticity spread at CP is often represented by several patterns; this implies "multilevel" (and functional) approach to botulinum therapy. In that case, the function may be improved only if BTA is simultaneously injected into the muscles belonging to several "levels" and spasticity patterns [17]. Appearance of modern instrumental method of objective motility evaluation, particularly, of computed and video gait analysis promoted development and standardization of the multilevel botulinum therapy based on identification of patterns.

Variants of classification of muscle spasticity patterns of both lower and upper extremities in patients with $\mathrm{CP}$ have been discussed in the foreign scientific literature numerous times $[9,14$, 16-18]. However, BTA dosage is still primarily calculated separately for each muscle, regardless of the total amount of drug per the extremity's functional segment; spasticity patterns have been described regardless of evaluation of their frequency rate at $\mathrm{CP}$ in general and at different clinical disease forms in particular. National scientific sources yield extremely scant information on the possibilities of using botulinum therapy for correction of muscle spasticity of upper extremities in children $[19,20]$. That is why we studied frequency rate of spasticity patterns in children with $\mathrm{CP}$ with hand lesion and evaluated spasticity reduction efficacy in upper extremities after the first BTA injections.

\section{Patients and methods}

The analysis involved results of observation of 52 patients, who had undergone treatment and rehabilitation at the FSBI "Scientific Center of Children's Health" in 2012-2013.

Trial inclusion criteria: 2-18 years of age, spastic CP forms with hand lesion, including CP with hyperkinetic component, no indications to the previous BTA injections in the muscles of upper extremities, no fixed contractures exceeding $15^{\circ}$ in the joints of upper extremities in patients.

We excluded from the trial patents with atonic-astatic CP form and prevalence of hyperkinetic motor disorders over spasticity; patients with fixed contractures in joints of upper extremities over $15^{\circ}$; the children, who underwent prescription or withdrawal of oral systemic antispasmodic drug within the given observation period.

Use of the BTA drug for the indication "muscle spasticity of upper extremities in children with CP", unregistered in the Russian Federation, was approved by the local Ethics Committee of the FSBI "SCCH"; we also received informed consent of parents / official representatives of the patients to BTA injections.

Age of patients (Me 25; 75\%) in boys was 4 years 2 months (2 years 10 months; 5 years 7 months), in girls -5 years 9 months (3 years 10 months; 10 years).

Diagnosis was established in compliance with the ICD-10 classification. Clinically, patients with 
spastic CP forms were divided into 2 groups: with unilateral and bilateral hand lesion. Total amount of patients with unilateral lesion -15 (28.9\%; 9 boys and 6 girls), with bilateral lesion 37 (71.1\%, 25 boys and 12 girls). Combination of spasticity and hyperkinesiae at CP was more often present in the bilateral hand lesion group (5 patients) than in the unilateral hand lesion group (1 patient).

All patients underwent standard, pediatric, neurological and orthopedic examination, including evaluation of muscular tonus, muscle strength and dynamic/fixed joint contractures. Muscular tonus evaluation was conducted on the basis of the Ashworth scale of spasticity [21].

On the basis of the published data $[9,14]$, we distinguished between 5 primary functionally significant segments determining motion capabilities of the whole upper extremity:

- shoulder joint (evaluation of muscles associated with flexion/extension, external and internal shoulder rotation, greater pectoral muscle);

- elbow joint (evaluation of forearm flexor and extensor muscles);

- forearm (primarily, evaluation of pronation depending on spasticity of round pronator and quadrate pronator muscles);

- radiocarpal joint (activity evaluation of flexor and extensor muscles of hands and fingers);

- fingers (emphasis on activity of thumb flexor and adductor muscles).

Spasticity was evaluated in the whole upper extremity's segment; this reflected clinically significant integrated tonus condition of all the segment's muscles before and after BTA injections and complex rehabilitation. We also evaluated muscle strength of each segment using a 6-grade muscle strength scale (Medical Research Council Weakness Scale, MRC, 1981).

We observed / kept video record of children's spontaneous motion and play activity in a pediatrician-psychologist's game room and gym in order to identify and describe the most typical patterns of motor disorders and spasticity in upper extremities' muscles.

We used botulinum toxin A drug Dysport (Ipsen Biopharm, Great Britain) (200 IU/ml (diluted)) for injections in spastic muscles of upper and lower extremities. Injections into muscles of upper extremities and profound muscles of lower extremities were conducted under ultrasound visualization control using an ultrasound diagnostic apparatus Accuvix V20 Prestige (Samsung Medison) with a linear sensor (frequency - 10-12 MHz) in the mode of visualization of musculoskeletal and superficial soft-tissue structures.

Calculating BTA dosage, we observed recommendations of the European consensus on the use of botulinum therapy for children with $\mathrm{CP}$ [22], according to which the safe dose of the drug is $1-20$ (25) IU/kg (according to data of several other studies, up to $30 \mathrm{IU} / \mathrm{kg}$ [23-25]), maximum total dose - 500-1,000 IU; maximum dose per injection site - 50-250 IU.

The drug's dose calculation for each target muscle for each injection was conducted on an individual basis given the child's age and weight, target muscle's volume and intensity of spasticity and general motor disorders.

Pathological patterns of spasticity and muscle strength were evaluated prior to BTA injection and 1 and 3 months after the conducted injections and rehabilitation in all 52 patients and 6 months after rehabilitation in $20(38.5 \%)$ patients. 7 (13.5\%) children received another BTA injection until the expiry of 6 months due to clinical indications.

Statistical analysis of the obtained data was conducted using program Statistica 6.1 (USA). All quantitative parameters were checked for normalcy of distribution with the help of KolmogorovSmirnov test. In case distribution was not normal, we used median $(\mathrm{Me})$ to describe central trend in the sample of quantitative and ordinal data; to describe measures of dispersion - minimal (min) and maximal $(\max )$ values and interquartile range. To describe qualitative and ordinal parameters, we analyzed absolute and relative parameter's frequencies in the group and expressed the obtained results in percentage of the total amount of values. To compare 2 independent groups of quantitative data, we used Mann-Whitney test; to compare 2 independent groups of qualitative data - Pearson and maximum likelihood $\chi^{2}$ test; to compare 1 quantitative or qualitative ordinal parameter in 2 dependent groups - Wilcoxon test. Critical significance 
level at check of statistical hypotheses was taken equal to 0.05 .

\section{Results}

\section{Spasticity and BTA dosage pattern at primary injections into upper and lower extremities' muscles.}

Injections into upper and lower extremities' muscles were conducted simultaneously. 11 (21.2\%) children received BTA injections only into upper extremities' muscles; 6 of them had hemiparesis, 5 - bilateral CP forms.

We distinguished the following pathological patterns of spasticity / pathological sets of upper extremities in children with spastic CP forms for the given sample of patients on the basis of evaluation of muscle strength and tonus, volume of active and passive movement in hands and daily motion activity: adduction and internal shoulder rotation, flexion in elbow joint, extension in elbow joint, forearm pronation, flexion in radiocarpal joint, flexion of fingers, thumb adduction and flexion. The typical patterns for lower extremities' muscles were adduction of thighs, rectus syndrome, flexion in knee joints, adduction and internal rotation of thighs, equinus deformity of feet, varus deformity of feet. Spasticity patterns of upper and lower extremities, the muscles associated with them and BTA dosages per 1 extremity are given in tb. 1 and 2.

Table 1. Distribution of BTA doses administered into upper extremities' muscles in patients with spastic CP forms.

\begin{tabular}{|c|c|c|c|c|c|c|}
\hline \multirow{2}{*}{\multicolumn{2}{|c|}{ Spasticity patterns and muscles }} & \multirow{3}{*}{$\begin{array}{c}\begin{array}{c}\text { Units of } \\
\text { measurement }\end{array} \\
\text { IU }\end{array}$} & \multicolumn{3}{|c|}{ BTA dose } & \multirow{3}{*}{$\begin{array}{c}\begin{array}{c}\text { Number of } \\
\text { muscles }\end{array} \\
29\end{array}$} \\
\hline & & & & & & \\
\hline \multirow[t]{2}{*}{$\begin{array}{l}\text { Shoulder adduction } \\
\text { and internal rotation }\end{array}$} & \multirow{2}{*}{$\begin{array}{l}\text { m. pectoralis major } \\
\text { m. subscapularis } \\
\text { m. latissimus dorsi }\end{array}$} & & 60 & $\begin{array}{l}30 \\
130\end{array}$ & $\begin{array}{l}40 \\
75\end{array}$ & \\
\hline & & $\mathrm{IU} / \mathrm{kg}$ & 3.4 & $\begin{array}{l}1.5 \\
4.3\end{array}$ & $\begin{array}{l}3.0 \\
4.0\end{array}$ & \\
\hline \multirow[t]{2}{*}{$\begin{array}{l}\text { Flexion in elbow } \\
\text { joint }\end{array}$} & \multirow{2}{*}{$\begin{array}{l}\text { m. biceps brachii } \\
\text { m. brachialis } \\
\text { m. brachioradialis }\end{array}$} & IU & 50 & $\begin{array}{l}20 \\
170\end{array}$ & $\begin{array}{l}40 \\
70\end{array}$ & \multirow[t]{2}{*}{55} \\
\hline & & $\mathrm{IU} / \mathrm{kg}$ & 3.0 & $\begin{array}{l}1.3 \\
6.3\end{array}$ & $\begin{array}{l}2.5 \\
3.9\end{array}$ & \\
\hline \multirow[t]{2}{*}{$\begin{array}{l}\text { Extension in elbow } \\
\text { joint }\end{array}$} & \multirow[t]{2}{*}{ m. triceps } & IU & 30 & $\begin{array}{l}30 \\
40\end{array}$ & $\begin{array}{l}30 \\
30\end{array}$ & \multirow[t]{2}{*}{6} \\
\hline & & $\mathrm{IU} / \mathrm{kg}$ & 2.1 & $\begin{array}{l}1.5 \\
2.9\end{array}$ & $\begin{array}{l}1.6 \\
2.7\end{array}$ & \\
\hline \multirow[t]{2}{*}{ Forearm's pronation } & \multirow[t]{2}{*}{$\begin{array}{l}\text { m. pronator teres } \\
\text { m. pronator quadratus }\end{array}$} & IU & 30 & $\begin{array}{l}15 \\
60 \\
\end{array}$ & $\begin{array}{l}30 \\
40\end{array}$ & \multirow[t]{2}{*}{71} \\
\hline & & $\mathrm{IU} / \mathrm{kg}$ & 2.0 & $\begin{array}{l}0.8 \\
4.3\end{array}$ & $\begin{array}{l}1.7 \\
2.5\end{array}$ & \\
\hline \multirow[t]{2}{*}{$\begin{array}{ll}\text { Flexion } & \text { in } \\
\text { radiocarpal joint }\end{array}$} & \multirow{2}{*}{$\begin{array}{l}\text { m. flexor carpi radialis } \\
\text { m. flexor carpi ulnaris } \\
\text { m. palmaris longus }\end{array}$} & IU & 30 & $\begin{array}{l}15 \\
70\end{array}$ & $\begin{array}{l}20 \\
60\end{array}$ & \multirow[t]{2}{*}{7} \\
\hline & & $\mathrm{IU} / \mathrm{kg}$ & 1.1 & $\begin{array}{l}0.5 \\
2.0\end{array}$ & $\begin{array}{l}1.0 \\
1.4\end{array}$ & \\
\hline \multirow[t]{2}{*}{ Flexion of fingers } & \multirow{2}{*}{$\begin{array}{lr}m . \quad \text { flexor } & \text { digitorum } \\
\text { profundus } & \text { et } \\
\text { superficialis } & \end{array}$} & IU & 80 & $\begin{array}{l}60 \\
140 \\
\end{array}$ & $\begin{array}{l}80 \\
100 \\
\end{array}$ & \multirow[t]{2}{*}{5} \\
\hline & & $\mathrm{IU} / \mathrm{kg}$ & 2.7 & $\begin{array}{l}2.2 \\
4.1 \\
\end{array}$ & $\begin{array}{c}2.7 \\
3.1 \\
\end{array}$ & \\
\hline \multirow{2}{*}{$\begin{array}{l}\text { Adduction and } \\
\text { flexion of thumb }\end{array}$} & \multirow[b]{2}{*}{$\begin{array}{l}\text { m. flexor pollicis longus } \\
\text { et brevis } \\
\text { m. adductor pollicis } \\
\text { brevis } \\
\text { m. interosseous I }\end{array}$} & IU & 10 & $5 ; 20$ & $5 ; 15$ & \multirow[t]{2}{*}{8} \\
\hline & & $\mathrm{IU} / \mathrm{kg}$ & 0.4 & $\begin{array}{l}0.2 \\
0.5\end{array}$ & $\begin{array}{l}0.3 \\
0.5\end{array}$ & \\
\hline
\end{tabular}


Table 2. Distribution of BTA doses administered into lower extremities' muscles in patients with spastic CP forms.

\begin{tabular}{|c|c|c|c|c|c|c|}
\hline \multirow{2}{*}{\multicolumn{2}{|c|}{ Spasticity patterns and muscles }} & \multirow{3}{*}{$\begin{array}{c}\begin{array}{c}\text { Units of } \\
\text { measurement }\end{array} \\
\text { IU }\end{array}$} & \multicolumn{3}{|c|}{ BTA dose } & \multirow{4}{*}{$\begin{array}{c}\begin{array}{c}\text { Number of } \\
\text { muscles }\end{array} \\
37\end{array}$} \\
\hline & & & $\mathrm{Me}$ & $\min$ & 25 & \\
\hline \multirow[t]{2}{*}{ Adduction of thighs } & \multirow{2}{*}{$\begin{array}{l}\text { m. adductor } \\
\text { magnus } \\
\text { m. adductor longus } \\
\text { m. adductor brevis }\end{array}$} & & 80 & $\begin{array}{l}40 \\
125\end{array}$ & $70 ; 90$ & \\
\hline & & $\mathrm{IU} / \mathrm{kg}$ & 5.2 & $\begin{array}{l}1.9 \\
8.1\end{array}$ & $\begin{array}{l}4.0 \\
7.0\end{array}$ & \\
\hline \multirow{2}{*}{ Rectus syndrome } & \multirow{2}{*}{ m. rectus } & IU & 45 & $30 ; 50$ & $35 ; 50$ & \multirow[t]{2}{*}{8} \\
\hline & & $\mathrm{IU} / \mathrm{kg}$ & 2.6 & $\begin{array}{c}2.1 \\
5.3\end{array}$ & $\begin{array}{c}2.5 \\
4.1\end{array}$ & \\
\hline \multirow[t]{2}{*}{ Flexion in knee joints } & \multirow{2}{*}{$\begin{array}{l}\text { m. semitendinosus } \\
\text { m. } \\
\text { semimembranosus }\end{array}$} & $\mathrm{IU}$ & 95 & $\begin{array}{l}60 \\
140\end{array}$ & $\begin{array}{l}80 \\
130\end{array}$ & \multirow[t]{2}{*}{10} \\
\hline & & $\mathrm{IU} / \mathrm{kg}$ & 4.7 & $\begin{array}{l}4.2 \\
9.1\end{array}$ & $\begin{array}{l}4.3 \\
7.3\end{array}$ & \\
\hline \multirow{2}{*}{$\begin{array}{l}\text { Adduction } \\
\text { internal rotation of } \\
\text { thighs }\end{array}$} & \multirow[t]{2}{*}{ m. gracilis } & $\mathrm{IU}$ & 60 & $\begin{array}{l}440 \\
100\end{array}$ & $40 ; 70$ & \multirow[t]{2}{*}{9} \\
\hline & & IU/kg & 3.3 & $\begin{array}{l}2.7 \\
5.7\end{array}$ & $\begin{array}{l}3.3 ; \\
3.9\end{array}$ & \\
\hline \multirow[t]{4}{*}{$\begin{array}{l}\text { Equinus deformity of } \\
\text { feet }\end{array}$} & \multirow[t]{2}{*}{ m. gastrocnemius } & IU & 100 & $\begin{array}{l}50 \\
350\end{array}$ & $\begin{array}{l}80 \\
160 \\
\end{array}$ & \multirow[t]{2}{*}{31} \\
\hline & & $\mathrm{IU} / \mathrm{kg}$ & 6.4 & $\begin{array}{l}2.1 \\
16.4\end{array}$ & $\begin{array}{l}4.8 \\
8.8\end{array}$ & \\
\hline & \multirow[t]{2}{*}{ m. soleus } & IU & 65 & $\begin{array}{l}40 \\
150\end{array}$ & $50 ; 80$ & \multirow[t]{2}{*}{9} \\
\hline & & $\mathrm{IU} / \mathrm{kg}$ & 3.5 & $\begin{array}{c}2.2 \\
5.0 \\
\end{array}$ & $\begin{array}{l}3.1 \\
3.9\end{array}$ & \\
\hline \multirow{2}{*}{$\begin{array}{l}\text { Varus deformity of } \\
\text { feet }\end{array}$} & \multirow{2}{*}{$\begin{array}{l}m . \\
\text { posterior }\end{array}$} & $\mathrm{IU}$ & 50 & $40 ; 70$ & $40 ; 65$ & \multirow[t]{2}{*}{4} \\
\hline & & $\mathrm{IU} / \mathrm{kg}$ & 3 & $\begin{array}{l}1.9 \\
3.6 \\
\end{array}$ & $\begin{array}{l}2.4 \\
3.3\end{array}$ & \\
\hline
\end{tabular}

The total dose of BTA injected to patients with CP (bilateral lesion) statistically significantly exceeded the total dose of BTA for patients with hemiparesis in absolute and relative terms (IU/kg). Neither absolute nor relative BTA doses injected into upper and lower extremities were significantly different in 2 clinical groups when calculating absolute and relative BTA dosage for each upper and lower extremity.

Pronator forearm set associated primarily with round pronator muscle was the most widespread (48 patients, $92.3 \%$ of the sample). The pronator set was most often combined with spasticity of forearm flexors; it was revealed in $12(80 \%)$ patients with hemiparesis and $32(86.5 \%)$ patients with bilateral $\mathrm{CP}$ forms. Flexion hand set was more often present in patients with hemiparesis than in patients with bilateral CP forms in the given sample of children [3 $(20 \%)$ cases to 3 $(8.1 \%)$ cases, $p=0.22]$. Share of children with flexion set of fingers and thumb adduction was also higher among patients with hemiparesis $[2(13.3 \%)$ to $2(5.4 \%), p=0.33 ; 3(20 \%)$ to 4 $(10.8 \%), \mathrm{p}=0.38]$; however, the difference was not statistically significant. Rate of spastic tension of greater pectoral muscles was higher in patients with bilateral CP forms [2 (13.4\%) to $14(37.8 \%), \mathrm{p}=0.02]$. Rate of hyperkinesiae was not significantly different in the 2 groups under study. Bilateral spasticity of round pronator muscles with no involvement of other muscles of "healthy" upper and lower extremities was observed in 2 patients with classic clinical hemiparesis presentation, anamnestic and instrumental data on the presence of perinatal intraventricular left-sided hemorrhage. Both patients were under 3 years of age.

Given the obtained functional results in upper extremities, the optimal BTA dosage for shoulder 
girdle muscles and forearm flexors at spastic $\mathrm{CP}$ forms in children was up to $4 \mathrm{IU} / \mathrm{kg}$ (for each functional segment), for triceps muscle of arm, forearm pronators and flexors of fingers - up to 3 $\mathrm{IU} / \mathrm{kg}$, for radiocarpal joint flexors - up to $1.5 \mathrm{IU} / \mathrm{kg}$ and up to $0.5 \mathrm{IU} / \mathrm{kg}$ for hand muscles.

Analysis of spasticity patterns and BTA doses for lower extremities' muscles showed that the dosage range (25-75\%) for greater muscles of thigh was 4-7 IU/kg; for gastrocnemius muscles up to $8.8 \mathrm{IU} / \mathrm{kg}(75 \%)$; for rectus, gracilis and salens muscles - up to $4 \mathrm{IU} / \mathrm{kg}(75 \%)$; for posterior tibial muscle - up to $3.3 \mathrm{IU} / \mathrm{kg}$.

\section{Spasticity changes in upper extremities in patients with spastic CP forms with hand lesion after BTA injections and complex rehabilitation}

The analysis involved only results of spasticity evaluation of the affected extremities, in which BTA was injected. Tonus was evaluated separately for each hand in case of tonus increase in both upper extremities in patients with bilateral CP forms and BTA injections on both sides. BTA was injected only in 1 extremity in $24(46.2 \%)$ children, in both upper extremities - in 28 (53.8\%); 80 hands in tote. Muscular tonus was evaluated in 52 patients after 3 months of observation (tb. 3) and in 20 patients after 6 months of observation on the individual basis.

Initially, muscular tonus in all the evaluated segments of upper extremities did not exceed 3 Ashworth scale grades. Muscular tonus was significantly decreasing within the first posttreatment month in both clinical groups. Positive tonus changes preserved until the $3^{\text {rd }}$ month of observation and could not be statistically detected 6 months after the first BTA injection. We observed spasticity increase in comparison with the initial level in a range of cases. We achieved the most significant muscular tonus reduction in forearm flexors and pronators at uni- and bilateral $\mathrm{CP}$ forms, in shoulder girdle muscles and hand flexors in patients with bilateral $\mathrm{CP}$ forms. Dynamics of spasticity recovery in hand muscles by the $6^{\text {th }}$ month of observation did not differ in patients with different clinical CP forms.

Table 3. Spasticity in upper extremities' muscles in 52 patients with spastic CP forms before, 1 and 3 months after botulinum therapy and complex rehabilitation.

\begin{tabular}{|c|c|c|c|c|c|c|c|}
\hline \multirow{2}{*}{$\begin{array}{c}\text { Upper } \\
\text { extremity's } \\
\text { segment }\end{array}$} & \multirow{2}{*}{$\begin{array}{l}\text { Ashworth } \\
\text { scale } \\
\text { grade }\end{array}$} & \multicolumn{3}{|c|}{$\begin{array}{c}\text { Unilateral CP forms, number of } \\
\text { extremities }(\%)\end{array}$} & \multicolumn{3}{|c|}{$\begin{array}{c}\text { Bilateral CP forms, number of } \\
\text { extremities }(\%)\end{array}$} \\
\hline & & $\begin{array}{l}\text { Before } \\
\text { BTA }\end{array}$ & 1 month & 3 months & $\begin{array}{l}\text { Before } \\
\text { BTA }\end{array}$ & 1 month & 3 months \\
\hline \multirow{7}{*}{$\begin{array}{l}\text { Shoulder } \\
\text { joint } \\
{ }^{*} \mathrm{p}_{0}=8 \times 10^{-6}\end{array}$} & $\mathbf{0}$ & $\begin{array}{c}13 \\
(86.6)\end{array}$ & $\begin{array}{c}15 \\
(100)\end{array}$ & $\begin{array}{c}14 \\
(93.3)\end{array}$ & $\begin{array}{c}12 \\
(18.5)\end{array}$ & $\begin{array}{c}16 \\
(24.6)\end{array}$ & $\begin{array}{c}17 \\
(26.2)\end{array}$ \\
\hline & 1 & $\begin{array}{c}1 \\
(6.7)\end{array}$ & 0 & $\begin{array}{c}1 \\
(6.7)\end{array}$ & $\begin{array}{c}26 \\
(40.0)\end{array}$ & $\begin{array}{c}45 \\
(69.3)\end{array}$ & $\begin{array}{c}44 \\
(67.7)\end{array}$ \\
\hline & 2 & $\begin{array}{c}1 \\
(6.7)\end{array}$ & 0 & 0 & $\begin{array}{c}21 \\
(32.3)\end{array}$ & $\begin{array}{c}4 \\
(6.1)\end{array}$ & $\begin{array}{c}4 \\
(6.1)\end{array}$ \\
\hline & 3 & 0 & 0 & 0 & $\begin{array}{c}6 \\
(9.2)\end{array}$ & 0 & 0 \\
\hline & 4 & 0 & 0 & 0 & 0 & 0 & 0 \\
\hline & & \multirow{2}{*}{\multicolumn{3}{|c|}{$\mathrm{p}<0.37$}} & \multicolumn{3}{|c|}{\begin{tabular}{l|l}
$*{ }^{*} \mathrm{p}_{0-1}<10^{-6}$ & $\mathrm{p}_{1-3}<0.56$ \\
\end{tabular}} \\
\hline & & & & & \multicolumn{3}{|c|}{$* \mathrm{p}_{0-3}<10^{-5}$} \\
\hline \multirow[t]{7}{*}{$\begin{array}{l}\text { Elbow joint } \\
\mathrm{p}_{0}=0.55\end{array}$} & $\mathbf{0}$ & 0 & $\begin{array}{c}5 \\
(33.3)\end{array}$ & $\begin{array}{c}5 \\
(33.3)\end{array}$ & $\begin{array}{c}5 \\
(7.7)\end{array}$ & $\begin{array}{c}17 \\
(26.2)\end{array}$ & $\begin{array}{c}15 \\
(23.0)\end{array}$ \\
\hline & 1 & $\begin{array}{c}3 \\
(20.0)\end{array}$ & $\begin{array}{c}9 \\
(60.0)\end{array}$ & $\begin{array}{c}6 \\
(40.0)\end{array}$ & $\begin{array}{l}13 \\
(20)\end{array}$ & $\begin{array}{c}38 \\
(58.4)\end{array}$ & $\begin{array}{c}25 \\
(38.5)\end{array}$ \\
\hline & 2 & $\begin{array}{c}11 \\
(73.3)\end{array}$ & $\begin{array}{c}1 \\
(6.7)\end{array}$ & $\begin{array}{c}4 \\
(26.7)\end{array}$ & $\begin{array}{c}38 \\
(58.5)\end{array}$ & $\begin{array}{c}10 \\
(15.4)\end{array}$ & $\begin{array}{c}25 \\
(38.5)\end{array}$ \\
\hline & 3 & $\begin{array}{c}1 \\
(6.7) \\
\end{array}$ & 0 & 0 & $\begin{array}{c}9 \\
(13.8) \\
\end{array}$ & 0 & 0 \\
\hline & 4 & 0 & 0 & 0 & 0 & 0 & 0 \\
\hline & & \multirow{2}{*}{\multicolumn{3}{|c|}{\begin{tabular}{r|r}
$* \mathrm{p}_{0-1}<0.0009$ & $\mathrm{p}$ \\
${ }^{*} \mathrm{p}_{0-3}<0.034$
\end{tabular}}} & $* \mathrm{p}_{0-1}<$ & & $1-3<0.01$ \\
\hline & & & & & \multicolumn{3}{|c|}{$* \mathrm{p}_{0-3}<3 \times 10^{-5}$} \\
\hline
\end{tabular}




\begin{tabular}{|c|c|c|c|c|c|c|c|}
\hline \multirow{7}{*}{$\begin{array}{l}\text { Forearm } \\
\text { (pronator } \\
\text { set) } \\
\mathrm{p}_{0}=0.7\end{array}$} & $\mathbf{0}$ & $\begin{array}{c}1 \\
(6.7)\end{array}$ & $\begin{array}{c}1 \\
(6.7)\end{array}$ & $\begin{array}{c}2 \\
(13.3)\end{array}$ & $\begin{array}{c}2 \\
(3.1)\end{array}$ & $\begin{array}{c}13 \\
(20.0)\end{array}$ & $\begin{array}{c}9 \\
(13.8)\end{array}$ \\
\hline & 1 & $\begin{array}{c}1 \\
(6.7)\end{array}$ & $\begin{array}{c}11 \\
(73.3)\end{array}$ & $\begin{array}{c}10 \\
(66.7)\end{array}$ & $\begin{array}{c}11 \\
(16.9)\end{array}$ & $\begin{array}{c}34 \\
(52.3)\end{array}$ & $\begin{array}{c}22 \\
(33.9)\end{array}$ \\
\hline & 2 & $\begin{array}{c}8 \\
(53.3)\end{array}$ & $\begin{array}{c}2 \\
(13.3)\end{array}$ & $\begin{array}{c}3 \\
(20.0)\end{array}$ & $\begin{array}{c}34 \\
(52.3)\end{array}$ & $\begin{array}{c}16 \\
(24.6)\end{array}$ & $\begin{array}{c}30 \\
(46.2)\end{array}$ \\
\hline & 3 & $\begin{array}{c}5 \\
(33.3)\end{array}$ & $\begin{array}{c}1 \\
(6.7)\end{array}$ & 0 & $\begin{array}{c}18 \\
(27.7)\end{array}$ & $\begin{array}{c}2 \\
(3.1)\end{array}$ & $\begin{array}{c}4 \\
(6.1)\end{array}$ \\
\hline & 4 & 0 & 0 & 0 & 0 & 0 & 0 \\
\hline & & \multicolumn{2}{|c|}{${ }^{*} \mathrm{p}_{0-1}<0.0009$} & $\mathrm{p}_{1-3}<0.65$ & ${ }^{*} \mathrm{p}_{0-1}$ & \multicolumn{2}{|c|}{\begin{tabular}{l|l} 
& $* \mathrm{p}_{1-3}<0.00065$ \\
\end{tabular}} \\
\hline & & \multicolumn{3}{|c|}{$* \mathrm{p}_{0-3}<0.02$} & \multicolumn{3}{|c|}{$* \mathrm{p}_{0-3}<2 \times 10^{-5}$} \\
\hline \multirow{7}{*}{$\begin{array}{l}\text { Radiocarpal } \\
\text { joint } \\
\mathrm{p}_{0}=0.14\end{array}$} & $\mathbf{0}$ & $\begin{array}{c}9 \\
(60.0)\end{array}$ & $\begin{array}{c}11 \\
(73.3)\end{array}$ & $\begin{array}{c}10 \\
(66.7)\end{array}$ & $\begin{array}{c}30 \\
(46.2)\end{array}$ & $\begin{array}{c}41 \\
(63.0)\end{array}$ & $\begin{array}{c}37 \\
(56.9)\end{array}$ \\
\hline & 1 & $\begin{array}{c}3 \\
(20.0)\end{array}$ & $\begin{array}{c}3 \\
(20.0) \\
\end{array}$ & $\begin{array}{c}3 \\
(20.0)\end{array}$ & $\begin{array}{c}29 \\
(44.6)\end{array}$ & $\begin{array}{c}22 \\
(33.9)\end{array}$ & $\begin{array}{c}22 \\
(33.9)\end{array}$ \\
\hline & 2 & $\begin{array}{c}3 \\
(20.0)\end{array}$ & $\begin{array}{c}1 \\
(6.7)\end{array}$ & $\begin{array}{c}2 \\
(13.3)\end{array}$ & $\begin{array}{c}4 \\
(6.1)\end{array}$ & $\begin{array}{c}2 \\
(3.1)\end{array}$ & $\begin{array}{c}3 \\
(4.6)\end{array}$ \\
\hline & 3 & 0 & 0 & 0 & $\begin{array}{c}2 \\
(3.1)\end{array}$ & 0 & $\begin{array}{c}3 \\
(4.6)\end{array}$ \\
\hline & 4 & 0 & 0 & 0 & 0 & 0 & 0 \\
\hline & & \multirow{2}{*}{\multicolumn{3}{|c|}{$\mathrm{p}<0.49$}} & $* \mathrm{p}_{0-1}<0$ & & $<0.32$ \\
\hline & & & & & \multicolumn{3}{|c|}{$* \mathrm{p}_{0-3}<0.00016$} \\
\hline \multirow[t]{6}{*}{$\begin{array}{l}\text { Fingers } \\
\mathrm{p}_{0}=0.09\end{array}$} & $\mathbf{0}$ & $\begin{array}{c}5 \\
(33.3)\end{array}$ & $\begin{array}{c}7 \\
(46.7)\end{array}$ & $\begin{array}{c}6 \\
(40.0)\end{array}$ & $\begin{array}{c}29 \\
(44.6)\end{array}$ & $\begin{array}{c}32 \\
(49.3)\end{array}$ & $\begin{array}{c}33 \\
(50.8)\end{array}$ \\
\hline & 1 & $\begin{array}{c}5 \\
(33.3)\end{array}$ & $\begin{array}{c}6 \\
(40.0) \\
\end{array}$ & $\begin{array}{c}8 \\
(53.3)\end{array}$ & $\begin{array}{c}30 \\
(46.2) \\
\end{array}$ & $\begin{array}{c}27 \\
(41.5) \\
\end{array}$ & $\begin{array}{c}25 \\
(38.5)\end{array}$ \\
\hline & 2 & $\begin{array}{c}4 \\
(26.7) \\
\end{array}$ & $\begin{array}{c}2 \\
(13.3) \\
\end{array}$ & $\begin{array}{c}1 \\
(6.7)\end{array}$ & $\begin{array}{c}4 \\
(6.1) \\
\end{array}$ & $\begin{array}{c}6 \\
(9.2) \\
\end{array}$ & $\begin{array}{c}7 \\
(10.7) \\
\end{array}$ \\
\hline & 3 & $\begin{array}{c}1 \\
(6.7)\end{array}$ & 0 & 0 & $\begin{array}{c}2 \\
(3.1)\end{array}$ & 0 & 0 \\
\hline & 4 & 0 & 0 & 0 & 0 & 0 & 0 \\
\hline & & \multicolumn{3}{|c|}{$\mathrm{p}<0.11$} & \multicolumn{3}{|c|}{$\mathrm{p}<0.14$} \\
\hline \multicolumn{2}{|c|}{$\begin{array}{c}\text { Total number of patients } \\
\text { (extremities) }\end{array}$} & \multicolumn{3}{|c|}{$15(15)$} & \multicolumn{3}{|c|}{37 (65) } \\
\hline
\end{tabular}

Note. Percentage of the total number of extremities in children with the clinical CP form.

* - significance level of differences $<0.05$;

$\mathrm{p}-$ significance level of spasticity differences before, 1 and 3 months after BTA injections and complex rehabilitation;

$\mathrm{p}_{0}$ - significance level of spasticity differences in patients with unilateral and bilateral lesion at CP before the BTA injection by segments;

$\mathrm{p}_{0-1}-$ significance level of spasticity differences before and 1 month after BTA injections and rehabilitation;

$\mathrm{p}_{1-3}-$ significance level of spasticity differences 1 and 3 months after BTA injections and rehabilitation;

$\mathrm{p}_{0-3}-$ significance level of spasticity differences before and 3 months 3 after BTA injections and rehabilitation.

Qualitative analysis of spasticity dynamics in hand muscles in 2 clinical groups after BTA injections and rehabilitation (pic. 1-5) revealed muscular tonus reduction down to 0-2 Ashworth scale grades for up to 3 months in shoulder girdle muscles, forearm flexors and muscles of fingers in patients with uni- and bilateral CP forms; this is an important factor of increase of motion volume in extremities and prevention of persistent articulomuscular contractures. We also managed to reduce the number of children with the highest ( 3 grades) spasticity in pronator and flexor muscles of hand and fingers in both clinical groups after BTA injection and rehabilitation (pic. 4, 5). In tote, according to the evaluation of all upper extremity segments, the share of children with minimal spasticity / normal muscular tonus (Ashworth scale) increased in both clinical groups by the $3^{\text {rd }}$ month of observation. 
Pic. 1. Ashworth scale spasticity distribution in shoulder girdle muscles before and after BTA injections in patients with uni- and bilateral lesion at $\mathrm{CP}$.

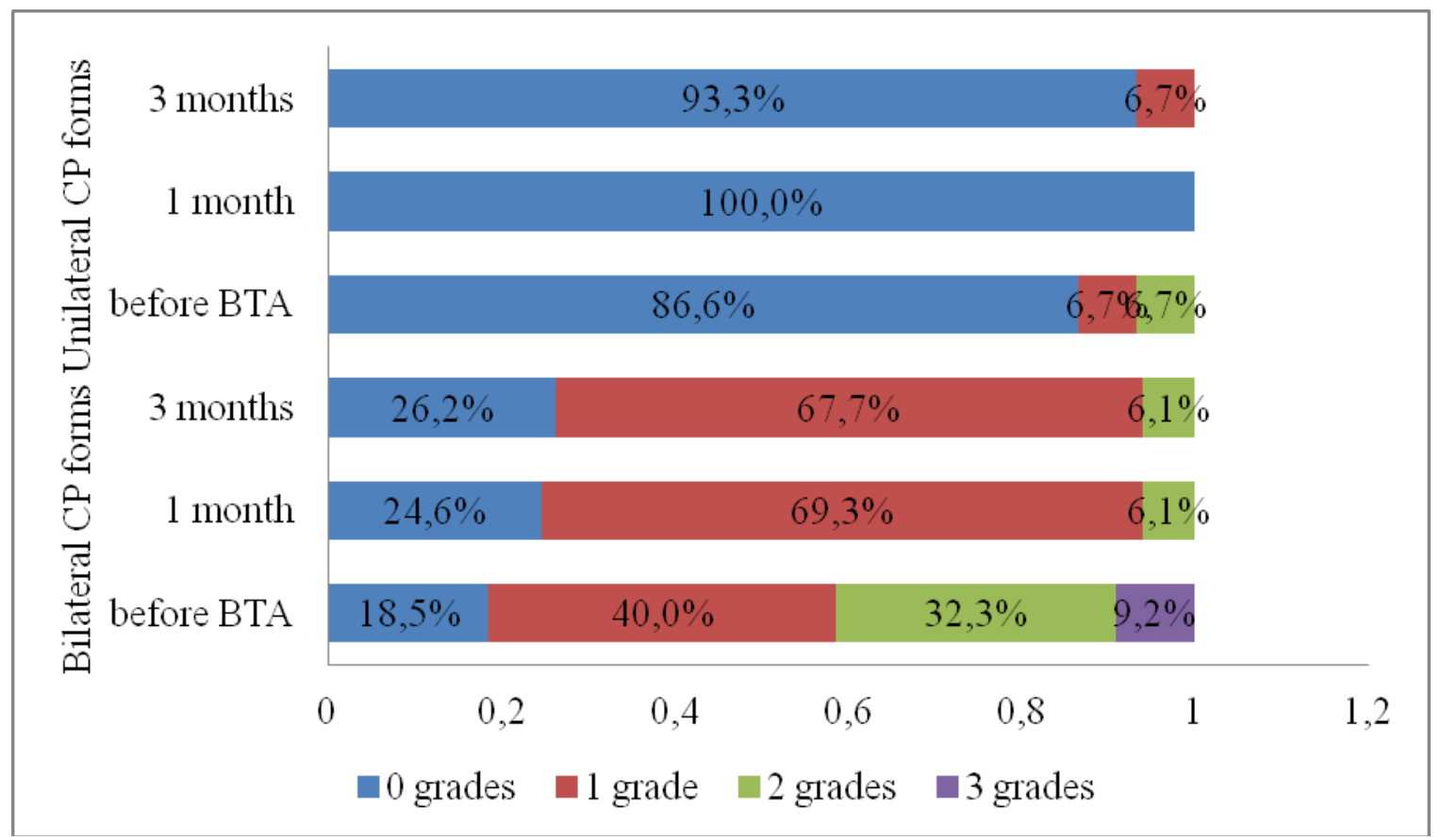

Pic. 2. Ashworth scale spasticity distribution in forearm flexor muscles before and after BTA injections in patients with uni- and bilateral lesion at $\mathrm{CP}$.

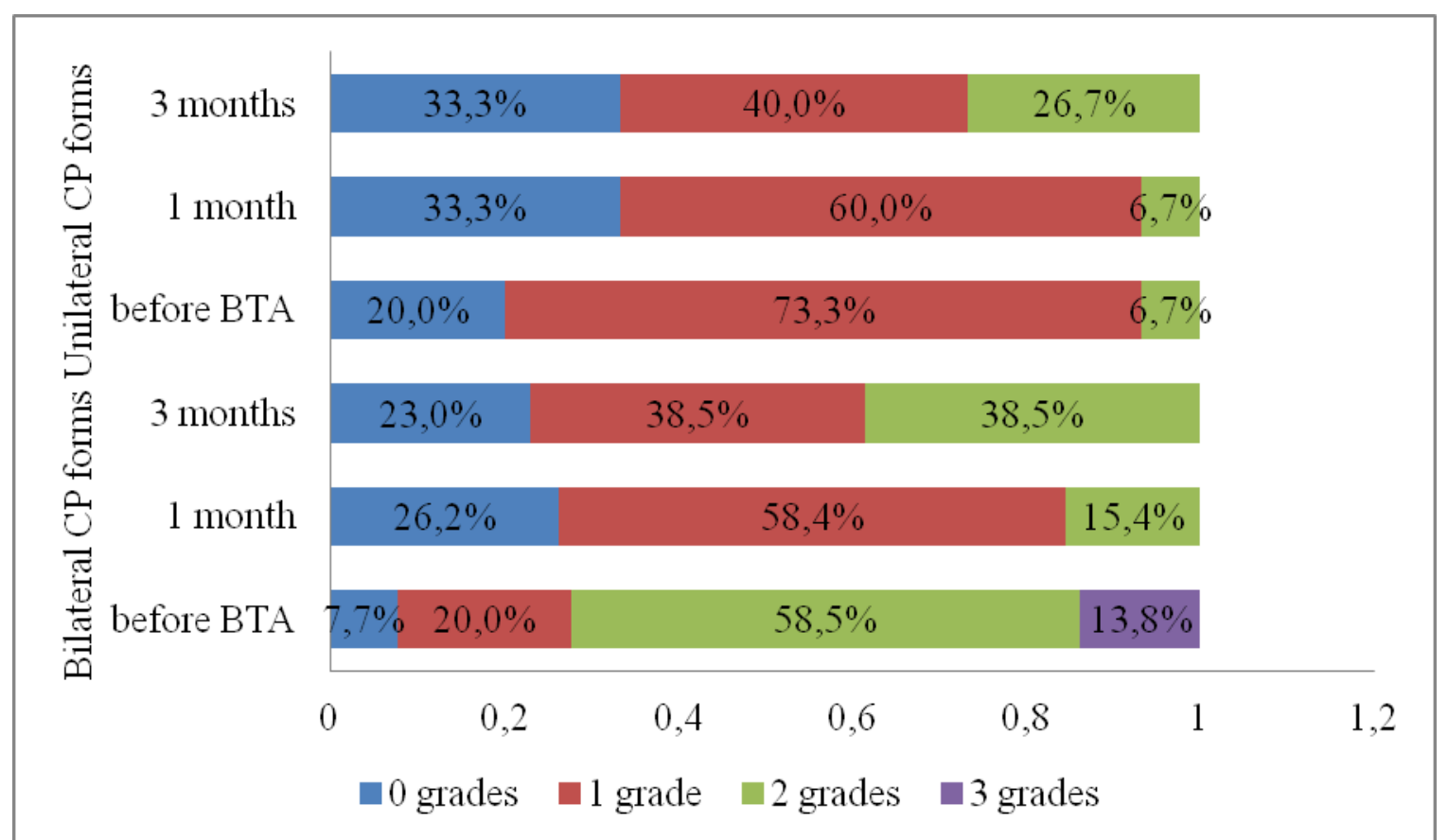

Pic. 3. Ashworth scale spasticity distribution in pronator muscles before and after BTA injections in patients with uni- and bilateral lesion at $\mathrm{CP}$. 


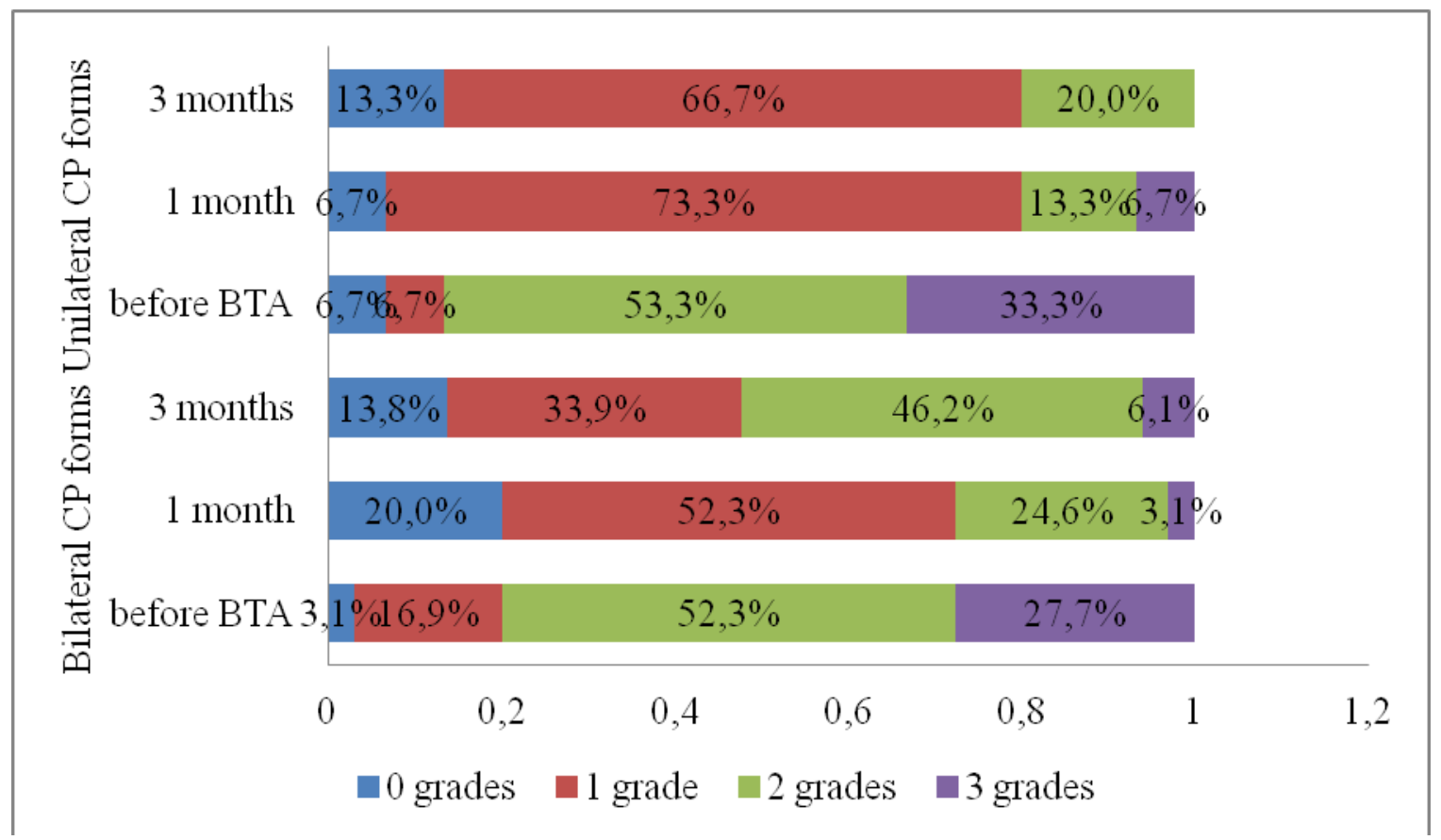

Pic. 4. Ashworth scale spasticity distribution in forearm muscles before and after BTA injections in patients with uni- and bilateral lesion at $\mathrm{CP}$.

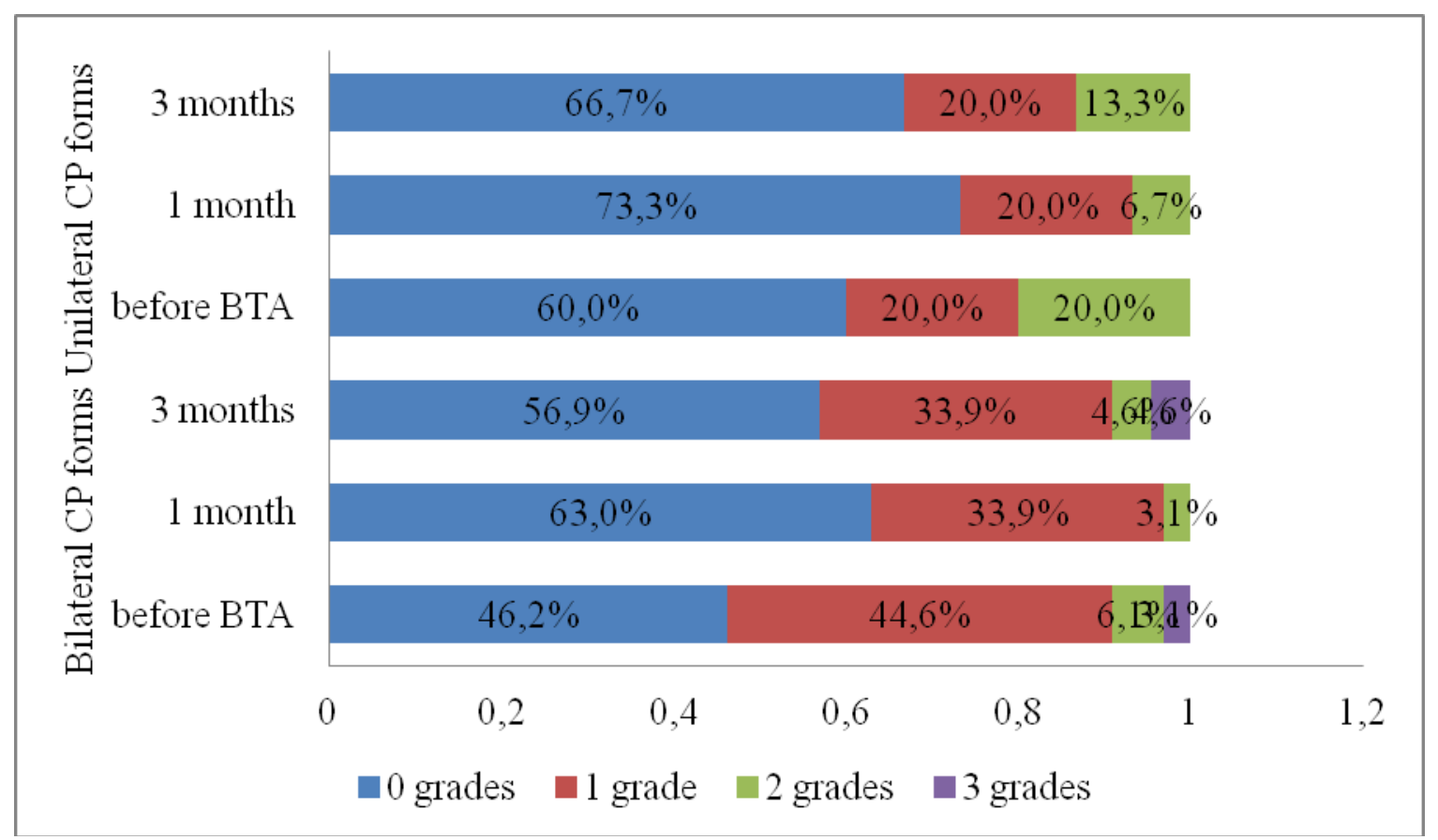

Pic. 5. Ashworth scale spasticity distribution in muscles of fingers before and after BTA injections in patients with uni- and bilateral lesion at $\mathrm{CP}$. 


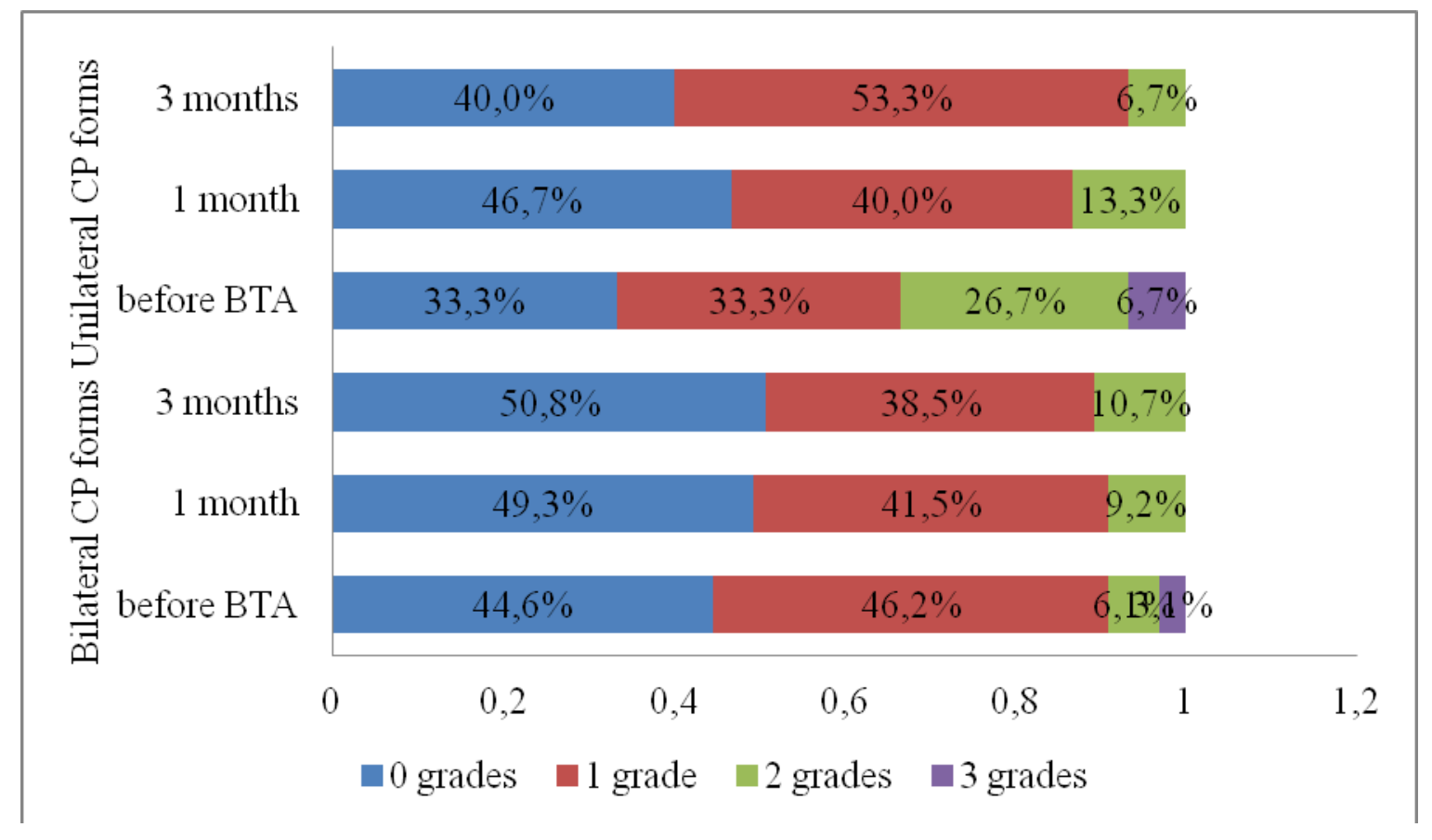

Botulinum therapy complications were registered in 6 cases $(11.5 \%)$ : we observed flaccidity, increased irritability and anorexia in 2 children within 2 post-injection days. In both cases the children were under 5 years of age and received BTA injections both in upper and lower extremities' muscles (total dose -24.5 and $28.3 \mathrm{IU} / \mathrm{kg}$ ) due to multilevel spasticity. The mentioned symptoms terminated themselves; the children began the planned rehabilitation period on the $30^{\text {th }}$ post-injection day. In the other 4 cases, side effects were associated with temporary strength reduction in the injected muscles. We observed adduction weakness and gait instability within 1.5 weeks after the BTA injection into adductors of thighs in the dosage of 4.4 $\mathrm{IU} / \mathrm{kg}$ on both sides in 1 patient; they normalized completely in the process of rehabilitation. We observed hand grip weakening by 1 MRC scale grade for 2 weeks in 3 patients after BTA injections into forearm muscles in the overall high doses $(5.1,3.7$ and $6.1 \mathrm{IU} / \mathrm{kg}$ per all muscles of 1 forearm); it returned to the initial level by the end of the $1^{\text {st }}$ month of observation. Clinically significant muscle strength reduction was not observed in any other cases.

\section{Discussion}

The primary issues of using BTA for treatment of spasticity of upper extremities' muscles in patients with $\mathrm{CP}$ are the selection of dosage and target muscles for injection. Lack of common opinion results in the situation, where all physicians using this method of treatment have to compile their own BTA usage protocols given the data of the latest clinical trials. We agree with most our colleagues, who rely on the analysis of spasticity patterns and combinations of functional disorders in children with CP for the selection of target muscles and BTA dosages [9, $14,18]$. This approach proved value at BTA injections into lower extremities' muscles [23] and may be used for treating spasticity of hand muscles.

The revealed prevalence of flexion-pronator spasticity pattern in upper extremities' muscles in patients with uni- and bilateral CP forms corresponds with the results obtained by other authors $[9,14]$, which also indicate a widespread combination of flexion and pronator sets. Another important point clinically and pathophysiologically is the pronounced prevalence of greater pectoral muscles' spasticity rate in patients with bilateral CP forms described in our work [2 $(13.4 \%)$ to $14(37.8 \%), p=0.02]$. Spasticity of greater pectoral muscles reflects pathological reflex activity [26, 27], which considerably affects posture maintenance and formation of gross motor skills in patients with diplegia and spastic tetraparesis. BTA injections in greater pectoral 
muscles in these cases allowed considerably reducing tonus for 3 post-injection months; this promoted not only the increase in the range of motion in upper extremities, but also quality improvement of general motor skills.

Round pronator muscle's spasticity revealed in almost all cases deserves special attention. Round pronator was one of the first muscles to have tonus increased in smaller patients with CP, before formation of "classic" flexion set; this corresponds with the observations [28]. Round pronator's increased tonus may indicate early spasticity of upper extremities' muscles. Round pronator tonus reduction due to BTA injections plays an important role not only in the prevention of contractures and forearm's active supination increase, but also, and more importantly, in preservation of hand grip strength due to the forearm's posterior surface muscles stretching reduction (with loss of muscle strength).

Tonus of flexor muscles prevails over tonus of extensors both in lower and upper extremities (as seen in the described group of patients with $\mathrm{CP}$ ); this results in the formation of primarily flexion spasticity patterns. Further research and increase in the volume of observations are required to clarify spasticity frequency of extensor muscles and their role in the formation of pathological movement pattern in patients with CP.

The described BTA dosages for injections in upper extremities' muscles are similar to the recommendations by A.S. Papavasiliou et al. (7.3 IU/ kg (4.7-11.4 IU/kg)) - 7.0, 1.0, $11.2(\mathrm{Me}$, min, max) IU/kg for patients with hemiparesis and 8.7, 2.4, 24.0 for patients with bilateral $\mathrm{CP}$ forms. The doses were reduced to 5.3, 1.1, 15.1., respectively, in dosage calculation per 1 upper extremity in patients with bilateral CP forms. Median doses for separate upper extremities' muscles were slightly lower in our study and usually did not exceed $4 \mathrm{IU} / \mathrm{kg}$ per 1 upper extremity's muscle segment in comparison with the recommendations by S. Berweck et al. (2-10 $\mathrm{IU} / \mathrm{kg}$ of BTA for smaller muscles and 10-30 IU/kg for greater muscles) [2].

As there were no significant differences between BTA dosages for hands of patients with uniand bilateral $\mathrm{CP}$ forms, we calculated Dysport dosage ranges for each upper extremity's segment:

- $0.3-0.5 \mathrm{IU} / \mathrm{kg}$ - for hand muscles;

- 2.7-3.1 IU $/ \mathrm{kg}$ - for flexors of fingers;

- $1.0-1.4 \mathrm{IU} / \mathrm{kg}$ - for radiocarpal joint flexors;

- $1.7-2.5 \mathrm{IU} / \mathrm{kg}$ - for forearm pronators;

- $1.6-2.7 \mathrm{IU} / \mathrm{kg}$ - for triceps muscle of arm;

- 2.5-3.9 IU $/ \mathrm{kg}$ - for forearm flexors;

- 3.0-4.0 IU $/ \mathrm{kg}$ - for shoulder girdle muscles.

Frequent combination of spasticity of both lower and upper extremities' muscles is fundamental to botulinum therapy. Tonus increase both in lower and upper extremities' muscles was observed in the $50(96 \%)$ described cases; BTA injections were made both in leg and hand muscles in 39 $(75.5 \%)$ cases. The need in single-step multilevel BTA administration to patients of low weight often leads to the drug's dosage reduction for each functional level or selection of priority muscles for botulinum therapy on that stage of the patient's motor development.

Evaluation of tonus dynamics in hand muscles in patients with $\mathrm{CP}$ after the primary singular BTA injections showed the presence of common trend in all the observed children - significant spasticity reduction and motion volume increase in joints 1 and 3 months after the injection and gradual muscular tonus increase by the $6^{\text {th }}$ month of observation; this corresponds with the results of other botulinum therapy efficacy observations both for lower and upper extremities' muscles [4, 28, 30, 31].

In several patients, spasticity exceeded the initial level by the end of the observation period or within 6 post-injection months. Spasticity increase in the period from month 3 to month 6 after the injection resulted in the need in the second BTA administration in $7(13.5 \%)$ cases. Given general tendencies of muscular tonus increase in upper extremities after singular botulinum therapy, control examination of patients 4 and 6 months after the injection and rehabilitation and the second BTA administration before muscular tonus restoration up to the initial level, though 
not earlier than 3 months after the previous BTA injections (in order to prevent formation of immunological resistance to the drug), appears reasonable.

The most frequent complication (4 children; 7.7\%) we observed in our trial was development of temporary weakness of hand grip after BTA injections in forearm muscles; according to most authors, who have studied this issue, this is the most widespread undesirable aftereffect of botulinum therapy of upper extremity muscles [32-35]. Excessive weakness may be prevented by applying modern methods of injection precision control and more concentrated solutions of the drug administered into hand muscles [36]. Ultrasound BTA injection precision control is recognized as the most prospective and convenient method of control in pediatric practice [18, 37-41]. In the given trial, ultrasound control was used at injections in forearm muscles, gracilis superficial leg muscles and posterior tibial muscles. Correct injection of the drug in the target muscles was confirmed not only by visual control, but also by clinical effect of injections in all cases; anatomically important injection-adjacent structures were not damaged in any case.

\section{Conclusions}

The most widespread spasticity pattern of upper extremities at cerebral palsy with hand lesion is forearm's pronator set caused by the round pronator muscle spasticity observed in $92.3 \%$ of patients, which is most often combined with spasticity of forearm flexor muscles. The main difference of pathological movement pattern of upper extremities in children with bilateral forms of cerebral palsy is higher rate of spastic tension of greater pectoral muscles $(37.8 \%)$ in comparison with children with hemiparesis (13.4\%).

Calculation of botulinum toxin A doses according to spasticity patterns of upper extremities' muscles allows minimizing amount of the injected drug and avoiding excessive muscular weakness in most cases.

Tonus of the upper extremities' muscles significantly decreases within the first post-treatment month, positive tonus changes remain until after 3 months of observation and cannot be statistically identified after 6 months in case of singular botulinum toxin A injections and complex rehabilitation in patients with uni- and bilateral forms of cerebral palsy.

\section{REFERENCES}

1. Koman L. A., Mooney J. F. 3rd, Smith B., Goodman A., Mulvaney T. Management of cerebral palsy with botulinum-A toxin: preliminary investigation. J Pediatr Orthop. 1993; 13 (4): 489-95.

2. Berweck S., Kirschner J., Heinen F. Therapy with botulinum toxin. In: Paediatric Neurology. Theory and practice (eds. Panteliadis C. P., Korinthenberg R.). Stuttgart; New York: Thieme. 2005. P. 925-951.

3. Delgado M. R., Hirtz D., Aisen M., Ashwal S., Fehlings D. L., McLaughlin J., Morrison L. A., Shrader M. W., Tilton A., Vargus-

Adams J. Quality Standards Subcommittee of the American Academy of Neurology and the Practice Committee of the Child Neurology Society. Practice parameter: pharmacologic treatment of spasticity in children and adolescents with cerebral palsy (an evidence-based review): report of the Quality Standards Subcommittee of the American Academy of Neurology and the Practice Committee of the Child Neurology Society. Neurology. 2010; 74 (4): 336-43.

4. Fehlings D., Novak I., Berweck S., Hoare B., Stott N. S., Russo R. N. Botulinum toxin assessment, intervention and followup for paediatric upper limb hypertonicity: international consensus statement. Eur J Neurol. 2010; 17 (Suppl. 2): 38-56.

5. Heinen F., Desloovere K., Schroeder A. S., Berweck S., Borggraefe I., van Campenhout A., Andersen G. L., Aydin R.,

Becher J. G., Bernert G., Caballero I. M., Carr L., Valayer E. C., Desiato M. T., Fairhurst C., Filipetti P., Hassink R. I., Hustedt U., Jozwiak M., Kocer S. I., Kolanowski E., Krageloh-Mann 
I., Kutlay S., Maenpaa H., Mall V., McArthur P., Morel E., Papavassiliou A., Pascual-Pascual I., Pedersen S. A., Plasschaert F. S., van der Ploeg I., Remy- Neris O., Renders A., Di Rosa G., Steinlin M., Tedroff K., Valls J. V., Viehweger E., Molenaers G. The updated European Consensus 2009 on the use of Botulinum toxin for children with cerebral palsy. Eur J Paediatr Neurol. 2010; 14 (1): 45-66.

6. Hoare B. J., Wallen M. A., Imms C., Villanueva E., Rawicki H. B., Carey L. Botulinum toxin $A$ as an adjunct to treatment in the management of the upper limb in children with spastic cerebral palsy (UPDATE). Cochrane Database Syst Rev. 2010; 1.

7. The official electronic resource of public health system in Australia Available at: http://www.medicareaustralia.gov.au/provider/pbs/drugs 1/botulinum.jsp.

8. Sakzewski L., Ziviani J., Boyd R. Systematic review and metaanalysis of therapeutic management of upper-limb dysfunction in children with congenital hemiplegia. Pediatrics. 2009; 123 (6): 1111-22.

9. Eliasson A. C., Burtner P. Improving Hand Function in Children with Cerebral Palsy. Mac Keith Press, 1 ed. 2008.

10. Eliasson A. C., Krumlinde-Sundholm L., Rosblad B., Beckung E., Arner M., Ohrvall A. M., Rosenbaum P. The Manual Ability

Classification System (MACS) for children with cerebral palsy: scale development and evidence of validity and reliability. Dev Med Child Neurol. 2006; 48 (7): 549-54.

11. Lam K., Lau K. K., So K. K., Tam C. K., Wu Y. M., Cheung G., Liang K. S., Yeung K. M., Lam K. Y., Yui S., Leung C. Can botulinum toxin decrease carer burden in long term care residents with upper limb spasticity? A randomized controlled study. J Am Med Dir Assoc. 2012; 13 (5): 477-84.

12. Palisano R., Rosenbaum P., Walter S., Russell D., Wood E., Galuppi B. Development and reliability of a system to classify gross motor function in children with cerebral palsy. Dev Med Child Neurol. 1997; 39 (4): 214-23.

13. Rodda J., Graham H. K. Classification of gait patterns in spastic hemiplegia and spastic diplegia: a basis for a management algorithm. Eur J Neurol. 2001; 8 (Suppl. 5): 98-108.

14. Miller F. Cerebral Palsy. New York: Springer Science. 2005. 1055 p.

15. Holmstrom L., Vollmer B., Tedroff K., Islam M., Persson J. K., Kits A., Forssberg H., Eliasson A. C. Hand function in relation to brain lesions and corticomotor-projection pattern in children with unilateral cerebral palsy. Dev Med Child Neurol. 2010; 52 (2): 145-52.

16. Ferrari A., Cioni G. The Spastic Forms of Cerebral Palsy. Italia: Springer-Verlag. 2010. 17. Molenaers G., Desloovere K., Fabry G., De Cock P. The effects of quantitative gait assessment and botulinum toxin a on mus culoskeletal surgery in children with cerebral palsy. $J$ Bone Joint Surg Am. 2006; 88 (1): 161-70.

18. Hustedt U. Botulinumtoxin bei spastischen Bewegungsstorungen. Ultraschallgestutzte Technik und Anwendung. KVM. 2010. pp. 13-15.

19. Umnov V. V., Novikov V. A. Travmatologiya i ortopediya Rossii - Traumatology and orthopedics in Russia. 2010; 1: 124-130.

20. Ashworth B. Preliminary trial of carisoprodal in multiple sclerosis. Practitioner. 1964; 192: 540-542.

21. Umnov V. V., Novikov V. A., Zvozil' A. V. Travmatologiya i ortopediya Rossii Traumatology and orthopedics in Russia. 2011; 3: 137-145.

22. Heinen F., Molenaers G., Fairhurst C., Carr L. J., Desloovere K., Chaleat Valayer E., Morel E., Papavassiliou A. S., Tedroff K., Ignacio Pascual-Pascual S., Bernert G., Berweck S., Di Rosa G., Kolanowski E., Krageloh-Mann I. European consensus table 2006 on botulinum toxin for children with cerebral palsy. Eur J Paediatr Neurol. 2006; 10 (5-6): 215-25.

23. Molenaers G., Schorkhuber V., Fagard K., Van Campenhout A., De Cat J., Pauwels P., Ortibus E., De Cock P., Desloovere K. Longterm use of botulinum toxin type A in children with cerebral palsy: treatment consistency. Eur J Paediatr Neurol. 2009; 13 (5): 421-9. 
24. Mall V., Heinen F., Siebel A., Bertram C., Hafkemeyer U., Wissel J. Treatment of adductor spasticity with BTX-A in children with CP: A randomized, double-blind, placebocontrolled study. Dev Med Child Neurol. 2006; 48 (1): 10-3.

25. Baker R., Jasinski M., Maciag-Tymecka I., Michalowska-Mrozek J., Bonikowski M., Carr L. Botulinum toxin treatment of

spasticity in diplegic cerebral palsy: a randomized, double-blind, placebo-controlled, dose ranging study. Dev Med Child Neurol. 2002; 44 (10): 666-75.

26. Semenova K. A., Mastyukova E. M., Smuglin M. Ya. Klinika i reabilitatsionnaya terapiya detskikh tserebral'nykh paralichei [Clinical Picture and Rehabilitation Therapy of Child Cerebral Palsy]. Moscow, Meditsina, 1973. 326 p.

27. Kislyakova E. A., Maslova N. N., Alimova I. L. Byulleten' sibirskoi meditsiny - Bulletin of Siberian medicine. 2008; 7 (3): 97-103.

28. Boyd R. N., Morris M. E., Graham H. K. Management of upper limb dysfunction in children with cerebral palsy: a systematic review. Eur J Neurol. 2001; 8 (Suppl. 5): 150-66.

29. Papavasiliou A. S., Nikaina I., Bouros P., Rizou I., Filiopoulos C. Botulinum toxin treatment in upper limb spasticity: treatment consistency. Eur J Paediatr Neurol. 2012; 16 (3): 237-42.

30. Zmanovskaya V. A. Klinicheskie varianty spasticheskikh form detskogo tserebral'nogo paralicha i otsenka effektivnosti botulinoterapii. Avtoref. dis. ... kand. med. nauk [Clinical Variants of Spastic Cerebral Palsy Forms and Evaluation of Botulinotherapy Effect. Author's abstract]. Ekaterinburg, 2011. 156 p.

31. Shen J., Ma J., Lee C, Smith B. P., Smith T. L. How muscles recover from paresis and atrophy after inframusculas injections of botulinum toxin A: study in juvenile rats. Journal of orthopedic research. 2006; 24 (5): 1128-1135.

32. Corry I. S., Cosgrove A. P., Walsh E. G., McClean D., Graham H. K. Botulinum toxin A in the hemiplegic upper limb: a double-blind trial. Dev Med Child Neurol. 1997; 39 (3): 185-93. 33. Fehlings D., Rang M., Glazier J., Steele C. An evaluation of botulinum-A toxin injections to improve upper extremity function in children with hemiplegic cerebral palsy. J Pediatr. 2000; 137 (3): 331-7.

34. Kawamura A., Campbell K., Lam-Damji S., Fehlings D. A randomized controlled trial comparing botulinum toxin A dosage in the upper extremity of children with spasticity. Dev Med Child Neurol. 2007; 49 (5): 331-7.

35. Russo R. N., Crotty M., Miller M. D., Murchland S., Flett P., Haan E. Upper-limb botulinum toxin A injection and occupational therapy in children with hemiplegic cerebral palsy identified from a population register: a single-blind, randomized, controlled trial. Pediatrics. 2007; 119 (5): 1149-58.

36. Klochkova O. A., Kurenkov A. L., Mamed"yarov A. M., Namazova-Baranova L. S., Gevorkyan A. K., Karimova Kh. M. Pediatricheskaya farmakologiya - Pediatric pharmacology. 2013; 10 (2): 80-86.

37. Schroeder A. S., Berweck S., Lee S. H., Heinen F. Botulinum toxin treatment of children with cerebral palsy - a short review of different injection techniques. Neurotox Res. 2006; 9 (23): 189-96.

38. Yang E. J., Rha D. W., Yoo J. K., Park E. S. Accuracy of manual needle placement for gastrocnemius muscle in children with cerebral palsy checked against ultrasonography. Arch Phys Med Rehabil. 2009; 90 (5): 741-4.

39. Berweck S., Feldkamp A., Francke A., Nehles J., Schwerin A., Heinen F. Sonographyguided injection of botulinum toxin A in children with cerebral palsy. Neuropediatrics. 2002; 33 (4): 221-3.

40. Westhoff B., Seller K., Wild A., Jaeger M., Krauspe R. Ultrasoundguided botulinum toxin injection technique for the iliopsoas muscle. Dev Med Child Neurol. 2003; 45 (12): 829-32. 41. Py A. G., Zein Addeen G., Perrier Y., Carlier R. Y., Picard A. Evaluation of the effectiveness of botulinum toxin injections in the lower limb muscles of children with cerebral palsy. 
Preliminary prospective study of the advantages of ultrasound guidance. Ann Phys Rehabil Med. 2009; 52 (3): 215-23. 